Original papers

\title{
Partial purification of $\alpha$-amylase from culture supernatant of Bacillus subtilis in aqueous two-phase systems
}

\author{
Wenbo Zhi $(\bowtie) \cdot$ Jiangnan Song $\cdot$ Jingxiu Bi $\cdot$ Fan Ouyang
}

W. Zhi $\cdot$ J. Song $\cdot$ J. Bi $\cdot$ F. Ouyang

National Key Laboratory of Biochemical Engineering, Institute of Process Engineering, Chinese Academy of Sciences, Beijing, 100080, People's Republic of China

\author{
W. Zhi \\ Fax: +86-10-82627061 \\ E-mail: zhiwenbo@sohu.com
}

Received: 15 November 2003 / Accepted: 30 June 2004

\begin{abstract}
A study was made of the partition and purification of $\alpha$-amylase from a culture supernatant of Bacillus subtilis in the polyethylene glycol (PEG) — citrate aqueous two-phase system (ATPS). Factors that influenced the partition of the protein in this system, including the molecular weight of the PEG, the tie line length of ATPS, the $\mathrm{pH}$ value and the sodium chloride concentration, were investigated. Purification of $\alpha$-amylase was attained with a purification factor (PF) of 1.8 and 90\% yield at $\mathrm{pH} 6.0$ in a PEG1000-citrate ATPS with short tie line length. By utilizing the salt-out effect of neutral salt, the purification of $\alpha$-amylase was further improved to 2.0 of PF and $80 \%$ yield in a PEG3350-citrate ATPS with $4 \%$ sodium chloride.
\end{abstract}

Keywords $\alpha$-Amylase $\cdot$ Aqueous two-phase systems $\cdot$ Purification

\section{Introduction}

Aqueous two-phase systems (ATPS) have been widely and successfully used in the extraction and purification of biological macromolecules, such as proteins [1, 2], nucleic acids [3] and antibiotics [4]. Compared with other traditional purification techniques, ATPS have various advantages, such 
as high water content of both phases $(70-80 \%, \mathrm{w} / \mathrm{w})$, high biocompatibility, low interfacial tension (minimizing degradation of biomolecules), high capacity and yield, ease of scale-up, and the possibility of polymer recycling [5].

The general properties of ATPS have been studied by several researchers $[5,6]$. However, the exact mechanism governing the partition of biomolecules is still not well understood. The partition coefficient for the biomolecule in an ATPS is influenced by van der Waals forces, hydrogen bonds, and hydrophobic and ionic interactions between the biomolecule and the surrounding phases. Therefore, compound separation is usually attained through the systematic variation of system compositions, including the type, molecular weight and concentration of the polymer, the type and concentration of the phase-forming salt, the $\mathrm{pH}$ value, the type and concentration of the neutral salts, and so on.

$\alpha$-Amylase is a commercially-important enzyme widely used in many industrial fields, including starch processing, brewage, and sugar refining [7]. $\alpha$-Amylase from Bacillus subtilis has specific advantages over $\alpha$-amylases from other sources, due to its improved thermo-stability and higher activity [8]. $\alpha$-Amylase from $B$. subtilis has a characteristic MW of $48 \mathrm{kDa}$ and an isoelectric point (pI) of around five. The optimal $\mathrm{pH}$ value is between 5.3 and 6.4.

For this work, we chose a polyethyelene glycol (PEG) citrate ATPS for the purification of $\alpha$-amylase because of the relatively large physicochemical difference between the upper and lower phases, the ease in forming phases, the relatively low costs in comparison to polymer-polymer ATPS, and the low environmental toxicity in comparison to phosphate-based ATPS [9]. Various factors that affect the partition of the protein and the purification efficiency are evaluated in order to maximize the activity, the purification factor (PF), and the recovery of $\alpha$-amylase.

\section{Materials and methods}

\subsection{Cell cultivation and production of $\alpha$-amylase}

A recombinant strain of B. subtilis BA-1 (a kind gift from Dr. Ming Gu, Institute of Process Engineering, Chinese Academy of Sciences, PR China) was grown in a $250 \mathrm{ml}$ shake flask with $20 \mathrm{ml}$ medium, containing $30.00 \mathrm{~g} / \mathrm{l}$ yeast extract, $6.99 \mathrm{~g} / \mathrm{l} \mathrm{Na}_{2} \mathrm{HPO}_{4} \cdot 12 \mathrm{H}_{2} \mathrm{O}, 4.00 \mathrm{~g} / 1 \mathrm{KH}_{2} \mathrm{PO}_{4}$, $5.24 \mathrm{~g} / 1 \mathrm{~K}_{2} \mathrm{HPO}_{4} \cdot 3 \mathrm{H}_{2} \mathrm{O}, 1.15 \mathrm{~g} / 1 \mathrm{NH}_{4} \mathrm{Cl}, 1.00 \mathrm{~g} / 1 \mathrm{MgSO}_{4} \cdot 7 \mathrm{H}_{2} \mathrm{O}, 0.05 \mathrm{~g} / 1 \mathrm{CaCl}_{2} \cdot 2 \mathrm{H}_{2} \mathrm{O}$, at $\mathrm{pH} 7.0$, a rotation speed of $220 \mathrm{rpm}$, and $37.0^{\circ} \mathrm{C}$. The supernatant was collected after $36 \mathrm{~h}$ cultivation by centrifugation at $9,000 \mathrm{~g}$ for $10 \mathrm{~min}$, and was stored at $4{ }^{\circ} \mathrm{C}$ for further use. 


\subsection{Determination of phase diagrams}

Binodal curves were determined according to the method of Alberttson [5]. Citrate solution of known concentration, composed of citric acid and sodium citrate mixed in a ratio that gives a $\mathrm{pH}$ value of 6.0, was added slowly to the concentrated PEG solution until the solution became turbid. The corresponding system composition was noted as a point close to the binodal curve, and $1.0 \mathrm{~g}$ of distilled water was then added, followed by shaking until the turbidity disappeared. The above steps were then repeated until enough points (around 10-15 points) were obtained to form a binodal curve.

\subsection{Preparation of aqueous two-phase systems}

Polyethylene glycol 400, in liquid form, was used directly—100\% (w/w). PEG1000, 3350 and 8000 were used in solid form. ATPS were prepared at room temperature by adding the required amount of PEG, sodium citrate, citric acid, $3 \mathrm{ml}$ culture supernatant and $200 \mu \mathrm{CaCl}_{2}(2.5 \mathrm{M})$, in $15 \mathrm{ml}$ graduated tubes with conical tips. Sodium chloride was added as required. The final weight was adjusted to $10 \mathrm{~g}$ by adding distilled water. After vortex mixing for $1 \mathrm{~min}$, phase separation was accelerated by centrifugation at $800 \mathrm{~g}$ for $3 \mathrm{~min}$.

\subsection{Assays}

$\alpha$-Amylase activity was measured using the starch-iodine method [10]. One unit of $\alpha$-amylase activity was defined as the amount of enzyme catalyzing the hydrolysis of $1.0 \mathrm{~g}$ soluble starch in $1 \mathrm{~h}$ at $\mathrm{pH} 6.0$ and $60^{\circ} \mathrm{C}$

Total protein concentration was measured by the method of Bradford [11]. The samples were read at $595 \mathrm{~nm}$ against the blanks with the same compositions as the samples, but without any proteins, to avoid the interference of PEG and citrate.

Two experiments were carried out in parallel for each measurement; the average values and the deviations (error bars for figures and absolute values for tables) of the results were calculated and recorded. 


\section{Results and discussion}

\subsection{Effect of phase-forming salt on $\alpha$-amylase activity}

The activity and stability of $\alpha$-amylase from $B$. subtilis largely depends on the calcium ion concentration [7]. However, the stable existence of calcium ions is hindered by the phase-forming salts commonly used in ATPS; the ions are precipitated by phosphate and chelated by citrate. Therefore, studies should be carried out to maintain the $\alpha$-amylase activity during the operation of ATPS, which usually lasts several hours. The effect of $\mathrm{CaCl}_{2}$ concentration on the maintenance of $\alpha$-amylase activity was evaluated and is shown in Fig. 1. The concentrations of phosphate and citrate were set at $20 \%(\mathrm{w} / \mathrm{w}) . \alpha$-Amylase activity decreased rapidly in the citrate system in the absence of $\mathrm{CaCl}_{2}$, which was due to $\mathrm{Ca}^{2+}$ elimination. But enzyme stability was enhanced when $\mathrm{Ca}^{2+}$ was added, and the deactivation rate of $\alpha$-amylase decreased slowly when the $\mathrm{CaCl}_{2}$ concentration was increased. When $0.56 \%(\mathrm{w} / \mathrm{w}) \mathrm{CaCl}_{2}$ was added to the citrate system, the deactivation rate of $\alpha$-amylase was even lower than that in the phosphate system. Under these conditions, $>80 \%$ of the enzyme activity was maintained for about $6.2 \mathrm{~h}$, more than threefold longer than in the $20 \%(\mathrm{w} / \mathrm{w})$ phosphate system. This is long enough for the ATP extraction operation and other related separation procedures, such as counter-current chromatography.

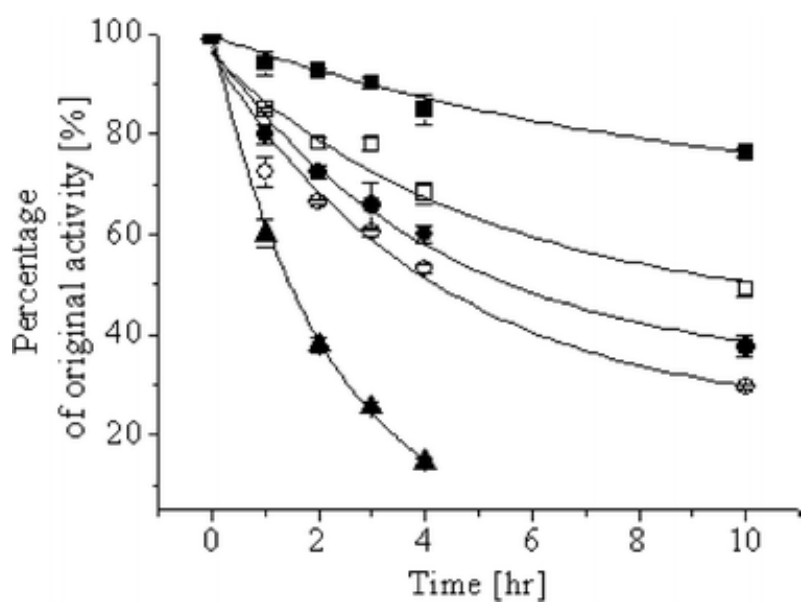

Fig. 1 Effect of different phase-forming salts and $\mathrm{CaCl}_{2}$ concentrations on the $\alpha$-amylase activity: (open squares) 20\% phosphate; (filled triangles) 20\% citrate; (open circles) $20 \%$ citrate $+0.11 \% \mathrm{CaCl}_{2}$; (filled circles) $20 \%$ citrate $+0.22 \% \mathrm{CaCl}_{2} ;$ (filled squares) $20 \%$ citrate $+0.56 \% \mathrm{CaCl}_{2}$ 


\subsection{Effect of PEG molecular weight and tie line length on a-amylase purification}

Since $\alpha$-amylase activity reaches its maximum at around $\mathrm{pH} 6.0$ and is stable over a relatively wide range of temperatures, all operations were carried out at $\mathrm{pH} 6.0$ and at room temperature $\left(20^{\circ} \mathrm{C}\right)$. As the citrate composition (the ratio of the citric acid to the sodium citrate) in the ATPS changes with $\mathrm{pH}$ value, which consequently affects the formation of the phases, we first determined phase diagrams for different PEG molecular weights at $\mathrm{pH}$ 6.0. The binodal curves corresponding to different PEG molecular weights are shown in Fig. 2. Above the binodal curves, ATPS is separated into two phases. Line $\mathrm{AB}$ is an example of the tie line for the evaluated system composition of PEG400. The points A and B correspond to the coordinates for the compositions of the upper and lower phases respectively.

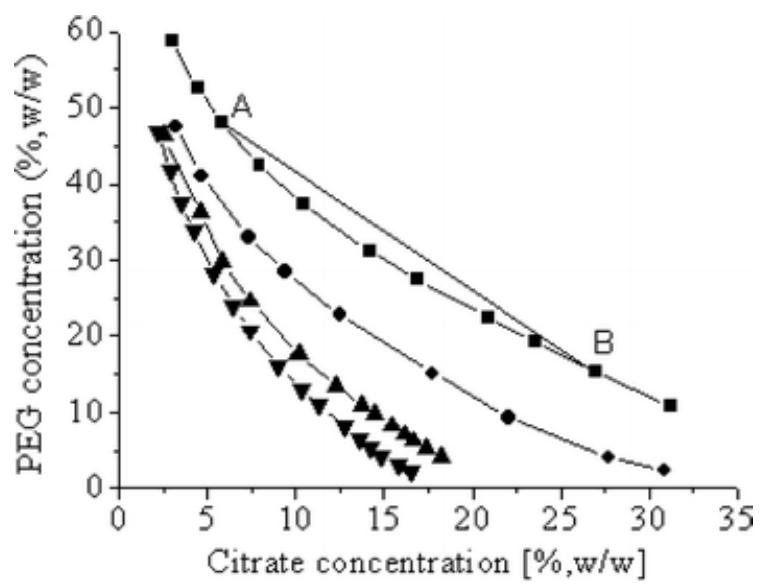

Fig. 2 Phase diagrams for PEG-citrate systems with different PEG molecular weights: (filled squares) PEG400; (filled circles) PEG1000; (filled triangles) PEG3350; (filled inverted triangles) PEG8000

The binodal curves become more asymmetric and closer to the origin as the molecular weight of the PEG is increased. Meanwhile, the physicochemical differences between the lower and upper phases become more significant and a lower concentration of PEG is required to form a phase.

To investigate the effects of the molecular weight of the PEG and the tie line length on the $\alpha$-amylase partition, several parameters, including phase volume ratio, partition coefficients, yield and PF were evaluated in ATPS with PEG molecular weights of 400, 1000, 3350 and 8000, respectively. For each PEG molecular weight, studies were carried out at three different ATPS compositions corresponding to three different tie line lengths. The results are shown in Table 1. The phase volume ratio is defined as the ratio of the upper phase volume to the lower phase volume; $K_{\mathrm{a}}$ and $K_{\mathrm{t}}$ are the partition coefficients of $\alpha$-amylase and total protein respectively, which are both 
defined as the ratio of the target protein concentration in the upper phase to that in the lower phase $\left(K=C_{\mathrm{U}} / C_{\mathrm{L}}\right)$; yield is defined as the percentage of target protein in the upper phase compared to the total target protein in the culture supernatant; PF is defined as the ratio of the $\alpha$-amylase yield to the total protein yield.

\section{[Table 1 will appear here. See end of document.]}

The data show that $K_{\mathrm{a}}$ and $K_{\mathrm{t}}$ both decrease with increasing PEG molecular weight, which agrees with the observations of Schmid et al [2] and Marcos et al [9]. As the degree of PEG polymerization increases, there are fewer hydroxyl groups for the same concentration of PEG, which leads to an increase in hydrophobicity in the PEG-richer upper phase. Meanwhile, an increase in PEG molecular weight results in less space for proteins to partition into the upper phase. Since $\alpha$-amylase has a comparatively high surface hydrophobicity, as evaluated by hydrophobic interaction chromatography [2], the size-exclusion effect is suggested to be the main reason for the decrease in the partition coefficients.

Although the decreasing trends for total protein and $\alpha$-amylase are similar, the decrease is steeper for the total protein, which means that more contaminant proteins are partitioned into the lower phase. Therefore, the best selectivity is achieved by PEG1000, as shown in Table 1.

The partitions of $\alpha$-amylase in ATPS with low molecular weight PEGs (PEG400 and 1000) were not significantly affected by tie line lengths, while for high molecular weight PEGs (PEG4000 and 8000), $\alpha$-amylase and total protein tended to partition into the lower phase as the tie line length increased, which may also be due to the size-exclusion effect caused by the relatively large size of PEG molecules with high molecular weight.

\subsection{Effect of $\mathrm{pH}$ on $\alpha$-amylase purification}

The investigation of effect of $\mathrm{pH}$ on the partition and purification was performed at $\mathrm{pH}$ values of 5.2, 6, 6.6 and 7.4 for three different tie line lengths in PEG1000-citrate systems (Fig. 3). The pH of ATPS was controlled by adjusting the ratio of sodium citrate to citric acid, which actually regulates the ratio of trivalent to divalent citrate ions. The phase-forming salt is totally composed of sodium citrate at $\mathrm{pH}$ 7.2. The tie line length for the same system composition (PEG and phase-forming salt concentration) decreased as the $\mathrm{pH}$ was lowered from 7.4 to 5.2, and the ATPS with a short tie line length did not even form two phases at a low $\mathrm{pH}$ of 5.2, which correlates with the idea that trivalent citrate ions are more effective for phase separation [9]. 

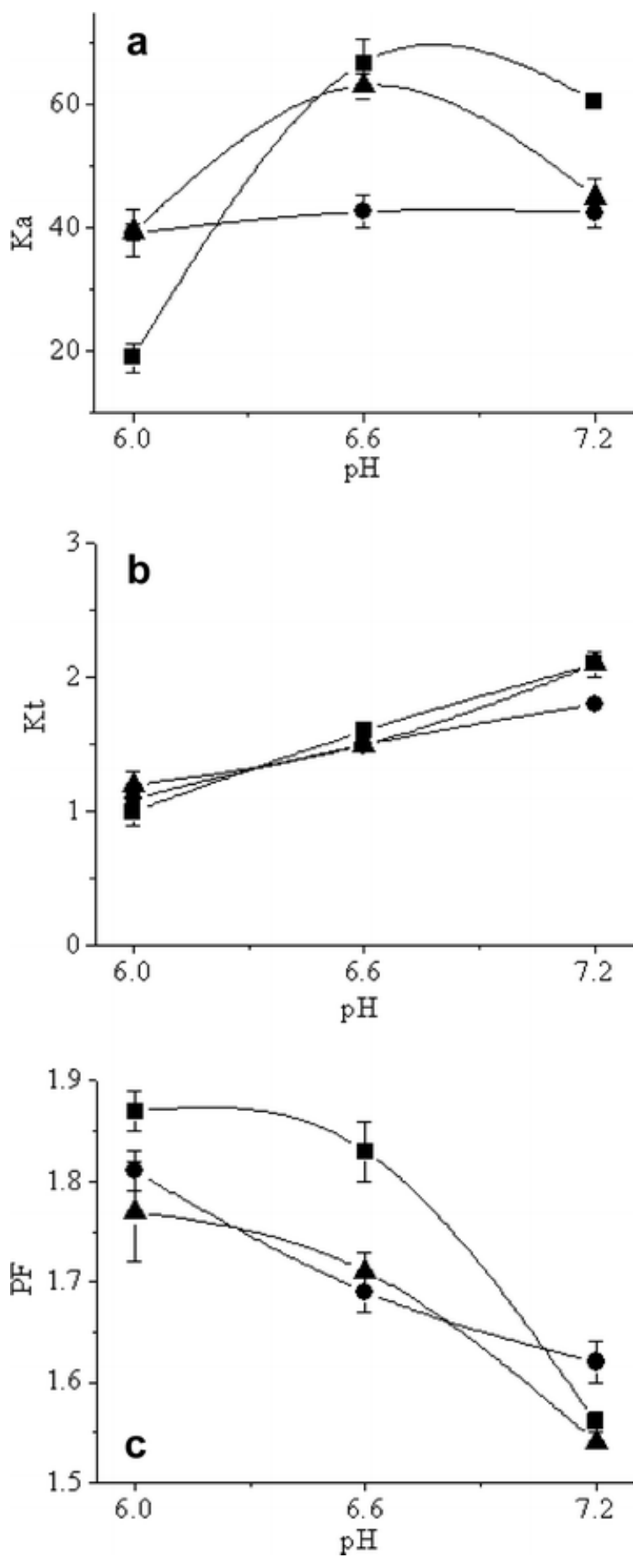

Fig. 3 Effect of $\mathrm{pH}$ on the partition coefficients of $\alpha$-amylase $\left(K_{\mathrm{a}}\right)$, total protein $\left(K_{\mathrm{t}}\right)$, and PF in PEG1000-citrate systems: (filled squares) short tie line length; (filled circles) medium tie line length; (filled triangle) long tie line length

The partition coefficients for $\alpha$-amylase $\left(K_{\mathrm{a}}\right)$ increase with $\mathrm{pH}$ for short and long tie line lengths and reach a maximum at around $\mathrm{pH} 6.6$, whereas the partition coefficient remained almost constant for a medium length tie line (Fig. 3a). The partition coefficients for total protein $\left(K_{\mathrm{t}}\right)$ also increased with $\mathrm{pH}$, although only to a very limited extent (Fig. 3b). The $\mathrm{pH}$ could affect protein partition by changing the solute charge in both phases. The $\mathrm{pI}$ of $\alpha$-amylase is around five, which is much 
lower than the effective $\mathrm{pH}$ we tested. Therefore, the negative charge of the $\alpha$-amylase increased as $\mathrm{pH}$ increased, resulting in a gradual distribution of the negatively charged $\alpha$-amylase to the upper phase [2]. However, as the $\mathrm{pH}$ was further increased, the total $\alpha$-amylase recovery from both phases decreased, which could be a reason that the partition coefficient of $\alpha$-amylase decreased to some extent at higher $\mathrm{pH}$.

The highest PF was attained at $\mathrm{pH} 6.0$, due to the interaction of $\alpha$-amylase and the total protein partition (Fig. 3c).

\subsection{Operating capacity}

The operating capacity of the PEG1000-citrate ATPS was investigated by testing the partition and purification of $\alpha$-amylase in PEG1000-citrate ATPS with three different tie line lengths after adding different amounts of culture supernatant. The results for the PF are shown in Fig. 4. Since the total weight of ATPS in the test tube is $10 \mathrm{~g}$, as described in the "Materials and methods" section, $\sim 6 \mathrm{~g}$ is around the greatest amount of supernatent that should be added to form ATPS besides PEG and phase-forming salts.

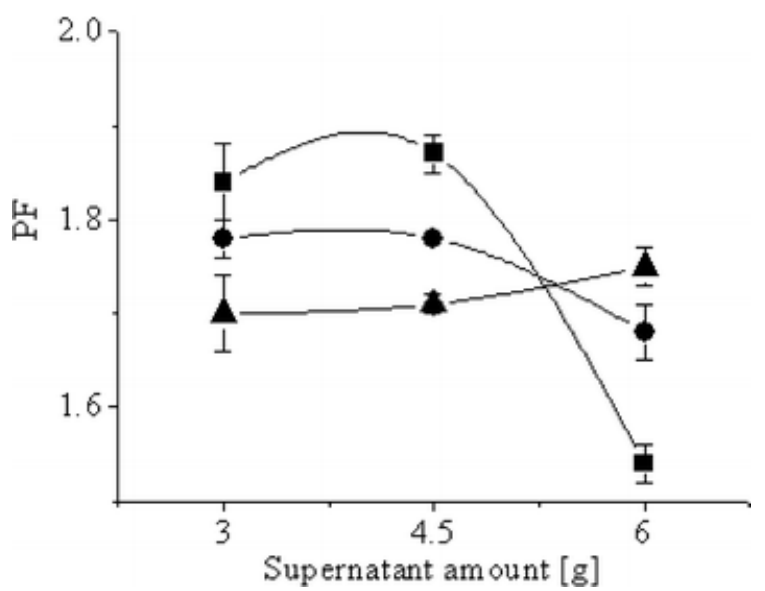

Fig. 4 Effect of amount of supernatant on the PF: (filled squares) short tie line length; (filled circles) medium tie line length; (filled triangles) long tie line length

As shown in Fig. 4, the PEG1000-citrate ATPS with short and medium tie line lengths were saturated, displaying an obvious decline in PF, when the amount of culture supernatant added exceeded $6 \mathrm{~g}$, whereas the PEG1000-citrate ATPS with a long tie line length was not saturated even after the maximum supernatant volume was added. However, due to its better separation and low viscosity, the PEG1000-citrate system with a short tie line and 3-4.5 g of supernatant is preferred. 


\section{$3.5 \mathrm{NaCl}$ addition}

The above results showed that higher PEG molecular weights (PEG3350 and PEG8000) resulted in lower partition coefficients for $\alpha$-amylase and total protein. In view of this, the partition of $\alpha$-amylase in PEG3350-citrate and PEG8000-citrate ATPS in the presence of $\mathrm{NaCl}$ was further investigated in order to improve the partition coefficient and possibly purification too (Fig. 5). The partition coefficients of both $\alpha$-amylase and total protein increased with the addition of $\mathrm{NaCl}$, although to different extents. This increase was more notable for $\alpha$-amylase than for total protein. The best selectivity was obtained in PEG3350-citrate ATPS in the presence of $4 \%(\mathrm{w} / \mathrm{w}) \mathrm{NaCl}$, with a PF of 2.0 (Fig. 4) and $80 \%$ yield (data not shown). The effect of $\mathrm{NaCl}$ addition on partition in PEG8000-citrate was similar to that on the partition in the PEG3350-citrate system, although a higher concentration of $\mathrm{NaCl}(8 \%)$ was needed to achieve a similar partition.

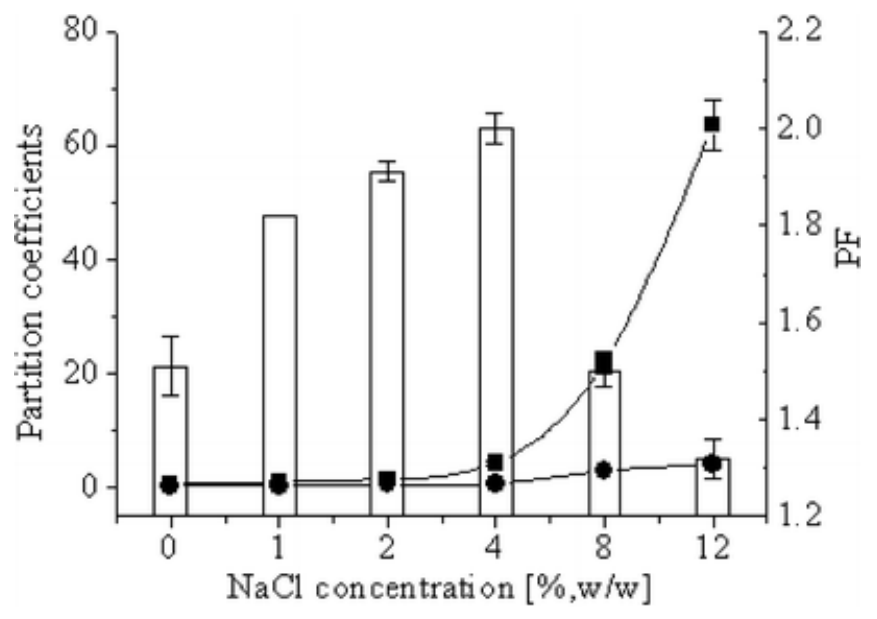

Fig. 5 Effect of $\mathrm{NaCl}$ concentration on the partition coefficients of $\alpha$-amylase $\left(K_{\mathrm{a}}\right)$, total protein $\left(K_{\mathrm{t}}\right)$ and PF: (filled squares) $K_{\mathrm{a}}$; (filled circles) $K_{\mathrm{t}}$; (open squares) PF

Since the hydrophobicities of the two phases are increased by adding $\mathrm{NaCl}$ to the PEG-salt systems, more hydrophobic proteins are prompted to partition into the upper phase. On the other hand, when the concentration of neutral salts is further increased and exceeds $1-5$ mol/l, proteins tend to partition into the upper phase due to the salt-out effect.

\section{Conclusions}

In this work, we investigated the effects of environmental conditions on $\alpha$-amylase purification in ATPS, including the system composition, the $\mathrm{pH}$ value and the $\mathrm{NaCl}$ concentration. Partial purification was attained, with a PF of 2.0 and $80 \%$ yield, in $11.0 \%$ PEG3350-14.0\% citrate ATPS 
with $4 \% \mathrm{NaCl}$ at $\mathrm{pH}$ 6.0. These results demonstrate the feasibility of single-step $\alpha$-amylase purification in PEG-citrate ATPS with low environmental toxicity.

Acknowledgements The authors would like to thank the support from the Chinese " 863 " National High Technology Grant-2002AA217031.

\section{References}

1. Diamond AD, Hsu JT (1992) Aqueous two-phase systems for biomolecule separation. Adv Biochem Eng Biot 47:89-135

2. Schmid AS, Ventom AM, Asenjo JA (1994) Partitioning and purification of $\alpha$-amylase in aqueous two-phase systems. Enzyme Microb Tech 16:131-142

3. Ohlsson R, Hentschel CC, Williams JG (1978) A rapid method for the isolation of circular DNA using an aqueous two-phase partition system. Nucleic Acids Res 5:583-590

4. Yang WY, Lin CD, Chu IM, Lee CJ (1994) Extraction of cephalosporin C from whole broth and separation of desacetyl cep halosporin C by aqueous two-phase partition. Biotechnol Bioeng 35:439-445

5. Alberttson PA (1986) Partition of cell particles and macromolecules, 3rd edn. Wiley, New York

6. Heriberto C (1996) Theory of phase formation in aqueous two-phase systems. J Chromatogr B 680:3-30

7. UpaDek H, Kottwits B (1997) Application of amylases in detergents. In: Van Ee JH, Misset O, Baas, E (eds) Enzymes in detergency. New York, pp 203-212

8. Saito N (1973) A thermophilic extracellular $\alpha$-amylase from Bacillus licheniformis. Arch Biochem Biophys 155:290-298

9. Marcos JC, Fonseca LP, Ramalho MT, Cabral JMS (1999) Partial purification of penicillin acylase from Escherichia coli in poly(ethylene glycol)-sodium citrate aqueous two-phase systems. J Chromatogr B 734:15-22

10. Yoo YJ, Hong J, Hatch RT (1987) Comparison of $\alpha$-amylase activities from different assay methods. Biotechnol Bioeng 30:147-151

11. Bradford MM (1972) A rapid and sensitive method for the quantification of microgram quantities of protein utilizing the principle of protein-dye binding. Anal Biochem 72:248 


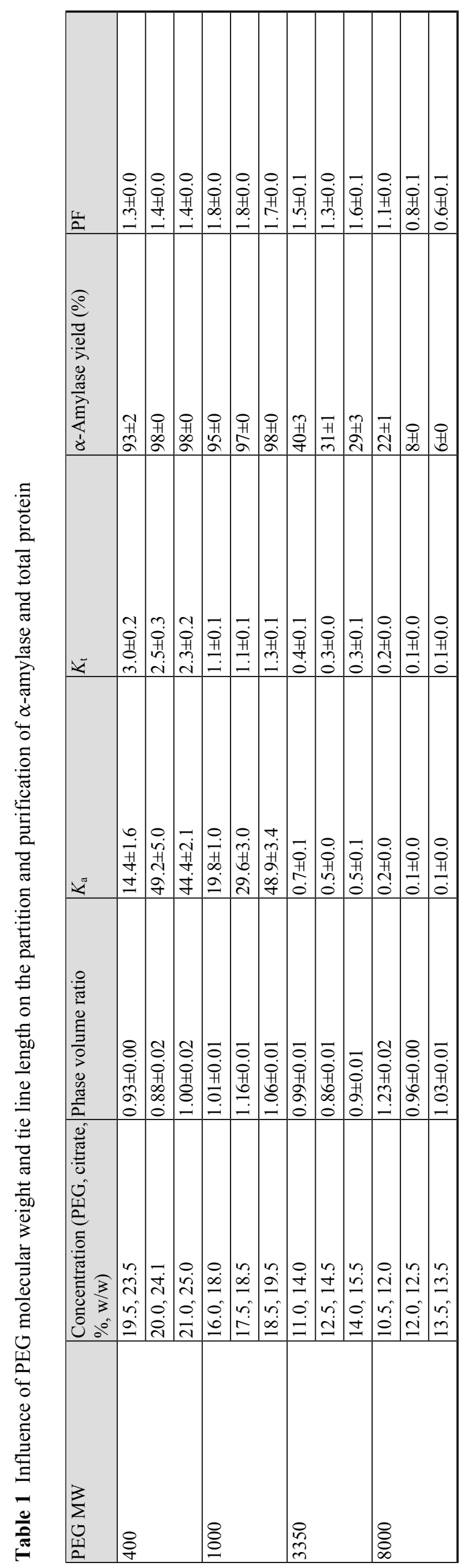

